\title{
Novel PTEN mutations in patients with Cowden disease: absence of clear genotype-phenotype correlations
}

\author{
Marcel R Nelen ${ }^{1}$, Hannie Kremer ${ }^{2}$, Irene BM Konings ${ }^{2}$, Frans Schoute ${ }^{2}$, \\ Anton J van Essen ${ }^{3}$, Rainer Koch ${ }^{4}$, C Geoffrey Woods ${ }^{5}$, Jean-Pierre Fryns ${ }^{6}$, \\ Ben Hamel $^{2}$, Lies H Hoefsloot ${ }^{2}$, Els AJ Peeters ${ }^{7}$ and George W Padberg ${ }^{1}$ \\ ${ }^{1}$ Department of Neurology, University Hospital Nijmegen \\ ${ }^{2}$ Department of Human Genetics, University Hospital Nijmegen, Nijmegen \\ ${ }^{3}$ Department of Medical Genetics, Groningen University, Groningen, The Netherlands \\ ${ }^{4}$ Department of Neurosurgery, Ruhr University Bochum, Bochum, Germany \\ ${ }^{5}$ Department of Clinical Genetics, St James's University Hospital, Leeds, UK \\ ${ }^{6}$ Center for Human Genetics, Leuven University, Leuven, Belgium \\ ${ }^{7}$ Department of Neurology, Leiden University Medical Center, Leiden, The Netherlands
}

\begin{abstract}
Cowden disease (CD) is characterised by multiple hamartomas in a variety of tissues. The pathological hallmark is the presence of a number of trichilemmomas. Several neurological symptoms are also part of CD with megalencephaly and Lhermitte-Duclos disease (LDD) as the most important features. Early recognition of $\mathrm{CD}$ patients is important because of the increased risk of developing malignancies. Breast cancer is the most frequent malignancy, but also urogenital, digestive tract, and thyroid cancers are found with higher frequencies. CD was localised to chromosome 10q23 and the PTEN gene (also known as MMAC1 or TEP1) was shown to be involved. Germline mutations were identified in both familial and sporadic CD patients. We identified eight PTEN mutations, of which seven were novel, in $13 \mathrm{CD}$ patients. Combined with previous data we have identified 17 independent CD mutations. Gross DNA alterations in CD patients were not detected. Genotype-phenotype relations are discussed. The only correlation suggested to exist is that missense mutations are not detected in LDD patients. However, larger numbers are needed to confirm this. Association of PTEN mutations and the occurrence of malignant breast disease found in an earlier study cannot be confirmed. Clinical features of five CD patients without a PTEN mutation in the coding sequence do not differ from CD patients with a PTEN mutation. Furthermore, it is likely that we have identified the majority of CD patients in the Netherlands. From this we estimate that CD has a prevalence of about 1 in 250000 in the Dutch population with a low mutation frequency.
\end{abstract}

Keywords: Cowden disease; multiple hamartoma syndrome; Lhermitte-Duclos disease; PTEN/MMAC1; tumour suppressor gene; chromosome 10

Correspondence: MR Nelen, Department of Neurology, University Hospital Nijmegen, PO Box 9101, 6500HB, Nijmegen, The Netherlands. Tel: + 3124 3615296; Fax: + 3124 3541122; E-mail: m.nelen@czzorlnm.azn.nl

Received 19 June 1998; revised 26 October 1998; accepted 29 October 1998 


\section{Introduction}

Cowden disease (CD, MIM No. 158350) is a hereditary autosomal dominant cancer syndrome with a marked variable expression, between and even within families. ${ }^{1,2}$ The main characteristic of this disorder is the formation of hamartomas in a variety of tissues. The majority of the patients (about 90\%) develop characteristic skin and mucocutaneous lesions. ${ }^{3-5}$ Multiple trichilemmomas, benign neoplasms of the hair follicle, are considered to be pathognomonic for this disease. ${ }^{6}$ Other typical features include oral and facial papules, goitre and benign hamartomas of the thyroid and digestive tract. ${ }^{7}$ A large number of $\mathrm{CD}$ patients (ie more than $80 \%$ ) develops macrocephaly, ${ }^{2}$ which is a true megalencephaly, but only a fraction of the patients develop Lhermitte-Duclos disease (LDD). This rare condition is believed to be a hamartomatous outgrowth of the cerebellum, characterised by hypertrophic ganglion cells. LDD was only recently identified as part of CD. ${ }^{8,9}$ Megalencephaly and LDD are the most important neurological features of $\mathrm{CD}$ and the cause of symptoms like tremor, ataxia, and mental retardation. $^{8-10}$ In addition to these benign symptoms, CD patients have an increased risk of developing malignancies. They include follicular cell carcinoma of the thyroid, carcinomas of the breast and malignant cancers of the urogenital and digestive tract. The highest risk is of breast cancer in females, approximately $70 \%{ }^{11}$

We assigned the gene responsible for CD to chromosome $10 \mathrm{q} 23$ by an extensive linkage study in 12 families. ${ }^{12}$ The gene encoding PTEN (alternatively named $M M A C 1$ or TEP1), a dual specificity phosphatase identified within the critical region, ${ }^{13-15}$ was shown to be involved by the identification of germline mutations present in a number of $\mathrm{CD}$ patients. ${ }^{16,17}$ Germline mutations were also identified in two other distinct but related syndromes, Bannayan-Riley-Ruvalcaba syndrome (BRR) and juvenile polyposis syndrome (JPS). ${ }^{18-21}$ Symptoms found in BRR and CD are partly overlapping and comprise macrocephaly, intestinal hamartomatous polyps, lipomas and haemangiomas. The R233X mutation in the PTEN gene has been found in both a CD and a BRR patient. ${ }^{19}$ This suggests that $\mathrm{CD}$ and BRR represent a variable spectrum of the same disorder. Alternatively, modifier genes might be responsible for the different phenotypes occurring in patients with an identical mutation. In JPS, characterised by hamartomatous polyps throughout the digestive tract and predisposition to digestive tract cancer, mutations have been identified which have so far not been described for $\mathrm{CD}$ or BRR patients. ${ }^{21}$ Genetic heterogeneity for JPS was suggested by a linkage study performed in eight informative JPS families. Linkage with the PTEN gene was excluded in these eight families and also mutation analysis of the coding region of the PTEN gene did not reveal any sequence alterations. ${ }^{22}$ Recently it was shown that the SMAD4 gene is the major gene involved in familial JPS. ${ }^{23}$

The identification of PTEN as a tumour suppressor has created a great deal of interest in the field of cancer research. Studies have shown the involvement of PTEN in a large number of sporadic tumours. Mutations have been identified in glioblastomas, prostate cancer, melanomas, thyroid and endometrial tumours. ${ }^{13,14,24-26}$ In some of these cancers, eg glioblastomas, melanomas and prostate cancer, PTEN mutations seem to be associated with tumour progression. ${ }^{24,27,28}$ In endometrial carcinomas PTEN turned out to be the most frequently mutated gene so far. ${ }^{26,29}$ Recent studies suggest that at least part of the function of PTEN is the regulation of cell migration and interactions with the extracellular matrix. ${ }^{30}$

Here we describe a detailed mutation analysis of the coding sequence of the PTEN gene in CD families and sporadic CD patients. We identified mutations in eight unrelated $\mathrm{CD}$ patients. One mutation was detected previously. Genotype-phenotype correlations are discussed for these eight mutations and nine mutations described previously. ${ }^{17}$

\section{Materials and Methods}

\section{Patients}

In total, 13 patients were included in the mutation analysis seven families and six sporadic patients. Dutch CD patients, both familial and sporadic, were diagnosed mainly on the presence of the dermatological lesions. ${ }^{2,6}$ Family anamnesis of the six sporadic patients was suspect in three cases. Parents of these patients were not examined for clinical signs. All patients fulfil the criteria of the international Cowden Consortium. ${ }^{12}$ Phenotypes of the $\mathrm{CD}$ patients and $\mathrm{CD}$ families with a PTEN mutation can be found in Table 1 . Of all patients combined, eight were diagnosed with LDD, four of which are familial. The five $\mathrm{CD}$ cases in which no PTEN mutation was detected were ascertained because of the presence of multiple features seen in CD. Family N1 has been used in the original linkage study and was previously described by Starink et al. ${ }^{2}$ Trichilemmomas, macrocephaly, and goitre were present in the proband analysed in CD family N9. The proband's mother died of thyroid carcinoma. Also her brother has clinical symptoms of CD. Patient 5899 has an extensive clinical history, including oral papillomatosis, goitre, endometrium carcinoma, and breast cancer. Patient n354 was ascertained because of the presence of skin lesions, goitre, 
Table 1 CD-mutations and their phenotype

\begin{tabular}{|c|c|c|c|c|c|c|c|c|c|c|c|}
\hline Patient & Sex & Skin & Thyroid & Breast & $I$ & $U G$ & $L D D$ & Head & Neurol. signs & \multicolumn{2}{|l|}{ Mutation } \\
\hline N6(F) & $3 \mathrm{f}$ & & & & n.e. & & & & & I33del, exon 2 & NT \\
\hline n331 & $\mathrm{f}$ & & & & & & & & & 159insTTAC, exon 2 & \\
\hline $\mathrm{N} 3(\mathrm{~F})$ & $2 \mathrm{~m} / 1 \mathrm{f}$ & & & & & & & & & IVS3+1G $>C$, intron 3 & \\
\hline $\mathrm{N} 4(\mathrm{~F})$ & $3 \mathrm{~m} / 2 \mathrm{f}$ & & & & n.e. & & & & & IVS4+1G $>\mathrm{T}$, intron 4 & \\
\hline $\mathrm{ddW}$ & $\mathrm{f}$ & & & & & & n.e. & & & IVS4+1G $>\mathrm{T}$, intron 4 & \\
\hline dd14443 & $\mathrm{f}$ & & & & & & & & & Q97X, exon 5 & \\
\hline $\mathrm{n} 40$ & $\mathrm{~m}$ & & & & n.e. & n.e. & & & & H123R, exon 5 & NT \\
\hline n130 & $\mathrm{f}$ & & & & & n.e. & & & & C124R, exon 5 & NT \\
\hline $\mathrm{N} 2(\mathrm{~F})$ & $2 \mathrm{~m} / 7 \mathrm{f}$ & & & & & & & & & R130X, exon 5 & \\
\hline $\mathrm{n} 264$ & $\mathrm{f}$ & & & & n.e. & n.e. & & & & R130X, exon 5 & \\
\hline $\mathrm{n} 275$ & $\mathrm{~m}$ & & & & & n.e. & & & & E157X, exon 5 & \\
\hline n342 & $\mathrm{m}$ & & & & & & & & & 545insA, exon 6 & \\
\hline $\mathrm{N} 8(\mathrm{~F})$ & $3 \mathrm{f}$ & & & & n.e. & & & & & G165E, exon 6 & NT \\
\hline $\mathrm{n} 269$ & $\mathrm{~m}$ & & & & n.e. & n.e. & & & & 783delGA, exon 7 & \\
\hline $\mathrm{N} 5(\mathrm{~F})$ & $1 \mathrm{~m} / 6 \mathrm{f}$ & & & & & & & & & IVS7+1G $>$ T, intron 7 & \\
\hline N7(F) & $6 \mathrm{~m} / 3 \mathrm{f}$ & & & & & & & & & 937delA, exon 8 & \\
\hline WvL & $\mathrm{f}$ & & & & & & & & & L320X, exon 8 & \\
\hline
\end{tabular}

F: familial; I: Intestine; UG: urogenital; m: male; f: female; n.e.: not examined; white bar: normal; grey bar: benign lesions present or (skin) trichilemmomas and/or oral papilomatosis or (head) macrocephaly; black bar: malignancies; NT: non truncating.

and mamma-carcinoma. Family anamnesis of both patients was suspect. The fifth patient (n1420) has skin lesions although no trichilemmomas were detected on pathological examination. Furthermore, this patient has macrocephaly and is known to have epileptic seizures. Family anamnesis was negative.

\section{Mutation Analysis}

Sequence analysis was performed on PCR products, using intronic primers designed to amplify the nine different exons and the corresponding exon/intron boundaries of the PTEN gene. ${ }^{13}$ Genomic DNA of patients was isolated using standard procedures. Products were gel purified using a PCR purification kit (Qiagen, Westburg, Reusden, The Netherlands).
Cycle sequencing was performed in the 9600 Thermal Cycler (ABI, Nieuwe Kerk ald Ysel, The Netherlands) with the dRhodamine dye terminator kit (ABI) for 28 cycles consisting of $10 \mathrm{~s}$ at $96^{\circ} \mathrm{C}, 5 \mathrm{~s}$ at $50^{\circ} \mathrm{C}$ and $4 \mathrm{~min}$ at $60^{\circ} \mathrm{C}$. Sequencing products were precipitated with $70 \%$ ethanol, $0.5 \mathrm{~mm} \mathrm{MgCl}_{2}$ and resuspended in $2.5 \mu \mathrm{l}$ loading buffer. The samples $(2 \mu \mathrm{l})$ were run and analysed on an ABI 377 automated sequencer.

A PTEN cDNA probe, encompassing the complete coding sequence and part of the $3^{\prime}$ and 5' UTR, was used to screen for the presence of gross DNA alterations in patients without an identified mutation. DNA $(7 \mu \mathrm{g})$ was digested with HinDIII and PstI, blotted to GenescreenPlus and hybridised with the PTEN cDNA probe. As a control probe PMP22 cDNA (17p11.2-p12) was used. 


\section{Control Analysis}

Identified mutations were confirmed in independently amplified PCR products. Each mutation was checked for its presence in the normal population by allele-specific oligonucleotide hybridisation. The appropriate exon was amplified in 50 unrelated unaffected individuals using genomic DNA. Allele-specific oligonucleotides of 14-16 bp were synthesised containing either the wild type or the mutant sequence. PCR products were dot-blotted on to GenescreenPlus. The oligonucleotides were labelled radioactively and hybridised in $5 \times \mathrm{SSPE} / 0.3 \%$ SDS at $30^{\circ} \mathrm{C}$ overnight. Blots were washed in $5 \times \mathrm{SSPE} / 0.3 \% \mathrm{SDS}$ at the proper temperature. Insertions and small deletions were tested as length polymorphisms by amplifying the exons in the presence of $\alpha-\left({ }^{32} \mathrm{P}\right) \mathrm{dCTP}$ and separating them on a $6.6 \%$ denaturing acrylamide gel.

\section{Results}

We have screened 13 patients diagnosed with $\mathrm{CD}$ for mutations in the coding region of the PTEN gene and eight mutations were identified (Table 2). All mutations were heterozygously present in genomic DNA. One mutation was detected previously. The patient group consists of six sporadic cases and seven familial cases for which the corresponding families were linked to 10q23. The mutations included two small deletions located in exon 2 and 8 . Three splice site mutations were located in introns 3,4 , and 7 . One missense mutation was found in exon 6 . Two nonsense mutations were detected, one in exon 5 and the second in exon 8 . All mutations identified in $\mathrm{CD}$ families were shown to cosegregate with the disorder. None of the detected mutations were present in 50 controls, excluding common polymorphisms, and all mutations were confirmed in independently amplified PCR fragments.

In three sporadic and two familial CD patients we did not identify a mutation. We were only able to analyse the PTEN gene in four of these patients for any gross alterations. Genomic DNA, digested with HinDIII and PstI, was hybridised with a PTEN cDNA probe. This probe contained the entire coding sequence

Table 2 Description of the identified CD mutations with their predicted effect

\begin{tabular}{lcc}
\hline Patient & Mutation & Predicted effect \\
\hline N6(F) & 97-99delATT & I33del \\
N3(F) & IVS3+1G $\rightarrow$ C & splice-site mutation \\
ddW & IVS4+1G $\rightarrow$ T & splice-site mutation \\
dd14443 & 289C $>$ T & Q97X \\
N8(F) & $494 \mathrm{G}>$ A & G165E \\
N5(F) & IVS7 $+1 \mathrm{G} \rightarrow$ T & splice-site mutation \\
N7(F) & $937 d e l A$ & frameshift, premature stop \\
WvL & $959 T>A$ & L320X \\
\hline
\end{tabular}

( $\mathrm{F}=$ family). and a small portion of the $3^{\prime}$ and $5^{\prime}$ UTR. There were no indications of any gross alterations in the PTEN gene of these four patients.

\section{Discussion}

Cowden disease is an autosomal dominant cancer syndrome, caused by mutations in the recently cloned tumour suppressor gene PTEN, located at 10q23.1. ${ }^{12,16,17}$ In this study we have performed an extensive mutation analysis in 13 unrelated patients, in which we detected eight mutations. Combined with our previous data ${ }^{17}$ we have identified 17 mutations (Figure 1) in 22 patients. Two mutations were detected twice in our patient group. Mutations included missense $(18 \%)$, nonsense $(29 \%)$ and splice-site mutations (24\%), small insertions (12\%) and deletions (18\%). The mutations were dispersed throughout the gene with a clustering in exon 5. The mutations and a summary of clinical data are presented in Table 1.

In total, we identified in seven of the nine familial $\mathrm{CD}$ cases a PTEN mutation. Family N1, in which we did not find a mutation, has been used in the original linkage study and the data are compatible with linkage to $10 \mathrm{q} 23 .^{12}$ However, since the size of this family is small the data are not statistically significant. Clinical symptoms include skin lesions, macrocephaly, mamacarcinoma and goitre. Recently, three papers described the presence of a processed pseudogene or homologue PTH $2,{ }^{31-33}$ located on chromosome 9. To exclude involvement of $P T H 2$ in this family we tested markers, including D9S165, D9S1878 and D9S15. These markers flank the interval containing this gene. Haplotype analysis indicated that the $\mathrm{PTH} 2$ locus is not linked to $\mathrm{CD}$ in this family (data not shown). So far, only the coding region of the PTEN gene is analysed for mutations and gross alterations are excluded. Mutations in the 5 ' regulatory regions, other regions that influence proper PTEN functioning or intronic mutations that create alternative splice sites might be present in this family. The second family (N9) without a detected mutation in PTEN does show the typical skin lesions, confirmed by pathological examination. Also, goitre and macrocephaly are present. The proband's mother died of a metastasised thyroid carcinoma. We performed mutation analysis in the proband and are now collecting blood samples of other family members to either prove or exclude involvement of the PTEN gene. 


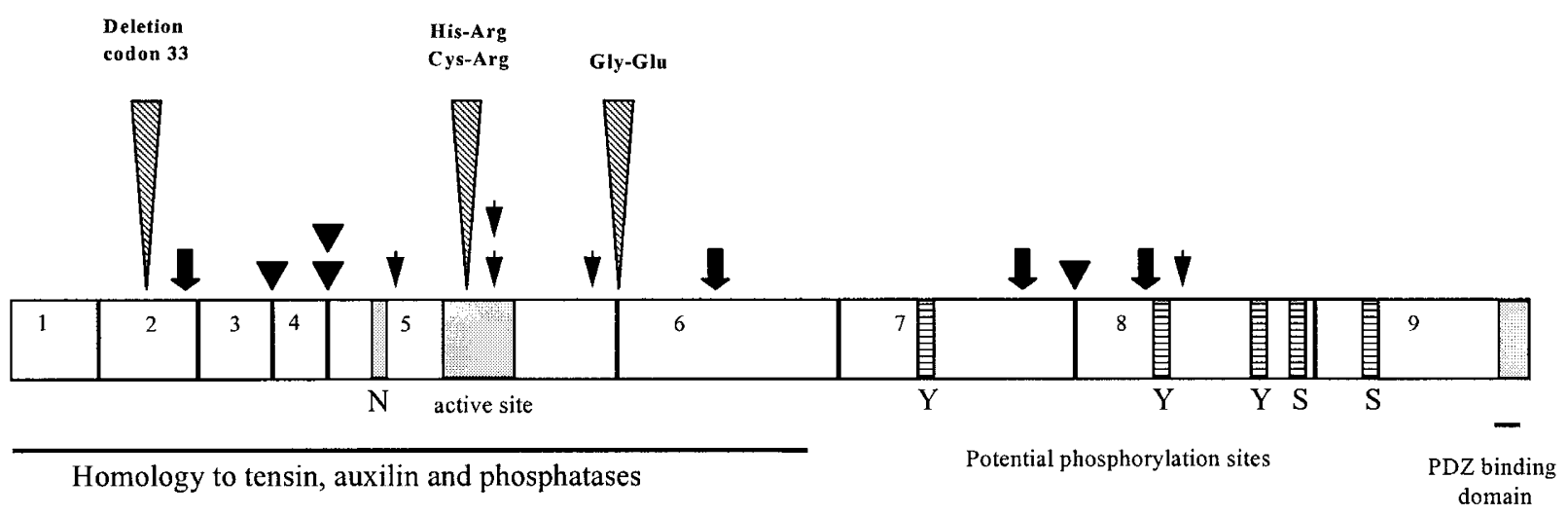

Insertion/deletion - premature stop
Nonsense mutation

Figure 1 Shown are the locations of the alterations detected in the PTEN gene in relation to the functional domains of the protein. Corresponding phenotypes are depicted in Table 1. The N-terminal part of PTEN is homologous to other phosphatases and also to tensin and auxilin. The C-terminal part of PTEN shares no homology with other proteins but contains three putative tyrosine (Y) and two putative serine phosphorylation $(S)$ sites and a PDZ binding domain at the $3^{\prime}$ end of the protein

In 10 of the 13 sporadic patients a mutation in the PTEN gene was detected. The three patients in which no PTEN mutation was detected had typical features of CD. These cases might have mutations in the same, not yet analysed, regions of the gene as mentioned above. Such mutations are not detectable by the methods used in this study. Another explanation for not finding mutations in both these sporadic and familial patients is genetic heterogeneity, suggested by the existence of CD families not linked to $10 \mathrm{q} 23.1 .{ }^{34}$ We also tested patients, offered to us for DNA diagnosis, with features commonly seen in CD but without the pathognomonic skin lesions. So far, we have not detected a mutation or a gross DNA alteration in any of these patients. The absence of mutations in this set of patients might underline the importance of skin lesions in $\mathrm{CD}$ diagnosis.

All but four mutations detected so far result in a premature truncation of the protein. This large proportion of protein truncating mutations is in agreement with the mutations described in the literature. ${ }^{20,35,36}$ Of the four mutations that do not result in a protein truncation, three are missense mutations and one is an in-frame 3 bp deletion. Two missense mutations result in a substitution of two invariant amino acids in the active site, His123 and Cys124, by an arginine. A dramatic decrease of phosphatase activity was shown for a His123Tyr substitution in the active site ${ }^{37}$ and one might expect this to be also true for these two missense mutations. The third missense mutation, Gly165Glu, involves the first amino acid residue of exon 6 . This mutation is localised in a structural element, an $\alpha$-helix, conserved between PTEN and tensin. Therefore, this mutation is predicted to disrupt the PTEN activity. ${ }^{38}$ The mutation is located at the second nucleotide in exon 6 at the splice acceptor site. However, it is unlikely that this mutation has a dramatic effect on RNA splicing since the second nucleotide of an exon is not part of the consensus sequence of the 3 ' splice site. ${ }^{39}$ The $3 \mathrm{bp}$ in-frame deletion (Ile33) does not result in protein truncation. Codon 33, however, is conserved in both tensin and auxilin. This mutation might affect interactions with other important molecules or the mutation might alter the stability of the RNA as is suggested by Lynch et al. ${ }^{20}$

The families and sporadic patients were considered for possible genotype-phenotype correlations. CD patients show involvement of multiple organ sites and all sites are involved in mutations throughout the protein. No specific mutation or part of the protein seems to be connected with a particular phenotype. We cannot exclude that there is a genotype-phenotype correlation for specific mutations. However, because only very few mutations are detected in more than one case a statistical analysis was not performed. Also, from family data we know that there is a marked variation in 
expression and age of occurrence of the various symptoms. This is in agreement with PTEN functioning as a tumour suppressor gene, as a second hit randomly occurs in different tissues. LDD, however, is not seen in patients with a missense mutation (eight cases). This is also true of the three LDD cases described in the literature. ${ }^{35}$ It is also notable that so far patients with mutations in exon 1, 8 and 9 have not been diagnosed with LDD. ${ }^{20,35}$ Furthermore, the two mutations found in exon 2 do not result in the typical papillary skin lesions. Only one of the three patients in family N6 has oral papillomatosis. A larger number of patients is needed to confirm any of these associations. We cannot confirm the observed association between the presence of a PTEN mutation and malignant breast disease. ${ }^{35}$ Four of the five female CD patients, of which two are mother and daughter, without a detected PTEN mutation do show malignancies of the breast. Furthermore, a brother of one of these female CD patients developed bilateral breast cancer. This is in contrast to the data reported in the literature, in which only one out of seven patients with no detected PTEN mutation had malignant breast disease. ${ }^{35}$ This indicates that larger numbers are needed to address these clinically relevant observations.

We have used the PALGA database, the Dutch network and database for pathology, to screen for any unknown CD patients. This network contains information of more than 4.5 million individuals. In total, we have identified $45 \mathrm{CD}$ patients in the age group of 25 years and older in the Dutch population, including patients not participating in this study. It is likely that we have identified the majority of patients in the Netherlands in this way. However, we cannot exclude possible misdiagnosis of CD patients. Although CD can be expressed with great variation, even within families, mutation analysis of both breast cancer patients and families indicates that the CD symptoms are highly expressed and well recognised by clinicians. Rarely is a PTEN mutation identified in such breast cancer cases. $^{40,41}$ Furthermore, CD can be attributed to a $P T E N$ mutation in the majority of $\mathrm{CD}$ patients. Therefore, we estimate that the prevalence of $\mathrm{CD}$ is between 1 in 200000 and 1 in 250000 in the Dutch population. In four patients it is likely that CD is caused by a new mutation. Three of the four cases are proven 'de novo' mutations. In two patients $(n 269, n 40)$ the expected mutation was not present in the parents and in family $\mathrm{N} 3$ the mutation could not be found in a healthy sister carrying the affected haplotype. This indicates a low mutation frequency, also shown by the rare finding of silent mutations in the coding sequence of $P T E N$ in the normal population.

The identification of in vivo PTEN substrates might reveal the cellular signalling pathways that are normally regulated by PTEN. This strategy might also identify protein kinases that counteract the effects of PTEN and are thus potentially oncogenic. A good candidate for such a kinase is the recently identified LKB1 gene, a serine threonine kinase, which is involved in Peutz-Jeghers syndrome. ${ }^{42}$ Patients are predisposed to the formation of intestinal hamartomatous polyps, mucocutaneous pigmentation affecting lips, buccal mucosa and digits and to many different types of cancer. It remains to be seen if both these tumour susceptibility genes function in the same pathways.

\section{Acknowledgements}

The authors would like to thank the families and patients for their participation in this study. We want to thank Dr E Mariman for helpful discussions. $\mathrm{MN}$ is supported by a grant from the Faculty of Medical Science, University of Nijmegen. HK is supported by the Dutch Cancer Society (KWF-KUN: 971386).

\section{References}

1 Loyd KM, Dennis M: Cowden's disease: a possible new symptom complex with multiple system involvement. Ann Intern Med 1963; 58: 136-142.

2 Starink TM, van der Veen JP, Arwert F et al: The Cowden syndrome: a clinical and genetic study in 21 patients. Clin Genet 1986; 29: 222-233.

3 Starink TM: Cowden's disease: analysis of fourteen new cases. J Am Acad Dermatol 1984; 11: 1127-1141.

4 Hanssen AM, Fryns JP: Cowden syndrome. J Med Genet 1995; 32: 117-119.

5 Longy M, Lacombe D: Cowden disease. Report of a family and review. Ann Genet 1996; 39: 35-42.

6 Starink TM, Meijer CJ, Brownstein MH: The cutaneous pathology of Cowden's disease: new findings. J Cutan Pathol 1985; 12: 83-93.

7 Brownstein MH, Mehregan AH, Bikowski JB, Lupulescu A, Patterson JC: The dermatopathology of Cowden's syndrome. Br J Dermatol 1979; 100: 667-673.

8 Padberg GW, Schot JD, Vielvoye GJ, Bots GT, de Beer FC: Lhermitte-Duclos disease and Cowden disease: a single phakomatosis [see comments]. Ann Neurol 1991; 29: 517-523.

9 Albrecht S, Haber RM, Goodman JC, Duvic M: Cowden syndrome and Lhermitte-Duclos disease. Cancer 1992; 70: 869-876. 
10 Eng C, Murday V, Seal S et al: Cowden syndrome and Lhermitte-Duclos disease in a family: a single genetic syndrome with pleiotropy? J Med Genet 1994; 31: 458-461.

11 Schrager CA, Schneider D, Gruener AC, Tsou HC, Peacocke M: Clinical and pathological features of breast disease in Cowden's syndrome: an underrecognized syndrome with an increased risk of breast cancer. Hum Pathol 1998; 29: 47-53.

12 Nelen MR, Padberg GW, Peeters EA et al: Localization of the gene for Cowden disease to chromosome 10q22-23. Nat Genet 1996; 13: 114-116.

13 Steck PA, Pershouse MA, Jasser SA et al: Identification of a candidate tumour suppressor gene, MMAC1, at chromosome $10 \mathrm{q} 23.3$ that is mutated in multiple advanced cancers. Nat Genet 1997; 15: 356-362.

$14 \mathrm{Li} \mathrm{J}$, Yen C, Liaw D et al: PTEN, a putative protein tyrosine phosphatase gene mutated in human brain, breast, and prostate cancer [see comments]. Science 1997; 275: 1943-1947.

15 Li DM, Sun H: TEP1, encoded by a candidate tumor suppressor locus, is a novel protein tyrosine phosphatase regulated by transforming growth factor beta. Cancer Res 1997; 57: 2124-2129.

16 Liaw D, Marsh DJ, Li J et al: Germline mutations of the PTEN gene in Cowden disease, an inherited breast and thyroid cancer syndrome. Nat Genet 1997; 16: 64-67.

17 Nelen MR, Van Staveren WCG, Peeters EA et al: Germline mutations in the pten/mmac1 gene in patients with Cowden disease. Hum Mol Genet 1997; 6: 1383-1387.

18 Arch EM, Goodman BK, Van Wesep RA et al: Deletion of PTEN in a patient with Bannayan-Riley-Ruvalcaba syndrome suggests allelism with Cowden disease. Am J Med Genet 1997; 71: 489-493.

19 Marsh DJ, Dahia PL, Zheng Z et al: Germline mutations in PTEN are present in Bannayan-Zonana syndrome [letter]. Nat Genet 1997; 16: 333-334.

20 Lynch ED, Ostermeyer EA, Lee MK et al: Inherited mutations in pten that are associated with breast cancer, cowden disease, and juvenile polyposis. Am J Hum Genet 1997; 61: 1254-1260.

21 Olschwang S, Serova Sinilnikova OM, Lenoir GM, Thomas G: Pten germ-line mutations in juvenile polyposis coli. Nat Genet 1998; 18: 12-14.

22 Marsh DJ, Roth S, Lunetta KL et al: Exclusion of PTEN and 10q22-24 as the susceptibility locus for juvenile polyposis syndrome. Cancer Res 1997; 57: 5017-5021.

23 Howe JR, Roth S, Ringold JC et al: Mutations in the smad4/dpc4 gene in juvenile polyposis. Science 1998; 280: 1086-1088.

24 Guldberg P, thor Straten P, Birck A, Ahrenkiel V, Kirkin $\mathrm{AF}$, Zeuthen J: Disruption of the MMAC1/PTEN gene by deletion or mutation is a frequent event in malignant melanoma. Cancer Res 1997; 57: 3660-3663.

25 Dahia PL, Marsh DJ, Zheng Z et al: Somatic deletions and mutations in the Cowden disease gene, PTEN, in sporadic thyroid tumors. Cancer Res 1997; 57: 4710-4713.

26 Risinger JI, Hayes AK, Berchuck A, Barrett JC: PTEN/ MMAC1 mutations in endometrial cancers. Cancer Res 1997; 57: 4736-4738.
27 Rasheed BK, Stenzel TT, McLendon RE et al: PTEN gene mutations are seen in high-grade but not in low-grade gliomas. Cancer Res 1997; 57: 4187-4190.

28 Suzuki H, Freije D, Nusskern DR et al: Interfocal heterogeneity of PTEN/MMAC1 gene alterations in multiple metastatic prostate cancer tissues. Cancer Res 1998; 58: 204-209.

29 Kong D, Suzuki A, Zou TT et al: PTEN1 is frequently mutated in primary endometrial carcinomas [letter]. Nat Genet 1997; 17: 143-144.

30 Tamura M, Gu J, Matsumoto K, Aota S, Parsons R, Yamakawa $\mathrm{H}$ : Inhibition of cell migration, spreading and focal adhesions by tumor suppressor PTEN. Science 1998; 280: $1614-1617$.

31 Kim SK, Su LK, Oh Y, Kemp BL, Hong WK, Mao L: Alterations of $\mathrm{pten} / \mathrm{mmacl}$, a candidate tumor suppressor gene, and its homologue, pth2, in small cell lung cancer cell lines. Oncogene 1998; 16: 89-93.

32 Teng DHF, Hu R, Lin H et al: Mmacl/pten mutations in primary tumor specimens and tumor cell lines. Cancer Res 1997; 57: 5221-5225.

33 Dahia PLM, FitzGerald MG, Zhang X et al: A highly conserved processed pten pseudogene is located on chromosome band 9p21. Oncogene 1998; 16: 2403-2406.

34 Tsou HC, Teng DH, Ping XL et al: The role of MMAC1 mutations in early-onset breast cancer: causative in association with Cowden syndrome and excluded in BRCA1-negative cases. Am J Hum Genet 1997; 61 1036-1043.

35 Marsh DJ, Coulon V, Lunetta KL et al: Mutation spectrum and genotype - phenotype analyses in Cowden disease and bannayan-zonana syndrome, two hamartoma syndromes with germline pten mutation. Hum Mol Genet 1998; 7: 507-515.

36 Rhei E, Kang L, Bogomolniy F et al: Mutation analysis of the putative tumor suppressor gene pten/mmacl in primary breast carcinomas. Cancer Res 1997; 57: 3657-3659.

37 Myers MP, Stolarov JP, Eng C et al: P-ten, the tumor suppressor from human chromosome 10q23, is a dualspecificity phosphatase. Proc Natl Acad Sci USA 1997; 94: 9052-9057.

38 Myers MP, Tonks NK: Pten: sometimes taking it off can be better than putting it on. Am J Hum Genet 1997; 61: 1234-1238.

39 Shapiro MB, Senapathy P: RNA splice junctions of different classes of eukaryotes: sequence statistics and functional implications in gene expression. Nucleic Acids Res 1987; 15: 7155-7174.

40 Ueda K, Nishijima M, Inui $\mathrm{H}$ et al: Infrequent mutations in the pten/mmacl gene among primary breast cancers. Jap J Cancer Res 1998; 89: 17-21.

41 Chen JD, Lindblom P, Lindblom A: A study of the pten/ mmacl gene in 136 breast cancer families. Hum Genet 1998; 102: 124-125.

42 Jenne DE, Reimann $\mathrm{H}$, Nezu J et al: Peutz-Jeghers syndrome is caused by mutations in a novel serine threonine kinase. Nat Genet 1998; 18: 38-44. 